\title{
Identification of cancer chemosensitivity by ODE and GMM modeling of heterogeneous cellular response to perturbations in fluorescent sphingolipid metabolism
}

\author{
Molina-Mora JA \\ Research Center on Tropical Diseases (CIET) \\ Faculty of Microbiology, University of Costa Rica (UCR) \\ San José, Costa Rica \\ jose.molinamora@ucr.ac.cr
}

\author{
Mora-Rodriguez RA \\ Research Center on Tropical Diseases (CIET) \\ Faculty of Microbiology, University of Costa Rica (UCR) \\ San José, Costa Rica \\ rodrigo.morarodriguez@ucr.ac.cr
}

\begin{abstract}
Cancer refers to a group of diseases in which cells display uncontrolled growth. Chemotherapy options are usually conditioned by generating resistance which is explained in part by the intrinsic heterogeneity of the population in the tumor. Because the study of resistance requires biosensors able to report the response at the single cell level, we propose to use fluorescently labeled SL to estimate pathway dynamics at the single cell level and investigate its possible application as gemcitabine (GMZ) response sensor in pancreatic cancer by comparing the dynamics between GMZ resistant (Panc-1, MiaPaca-2) and sensitive (BxPC3) cell lines. We used imaging flow cytometry to extract multiple image features and classify cells using gaussian-mixture model (GMM) to generate response fingerprints upon known perturbations. The results suggest that GMZ inhibits the Sphingomyelin-Synthase of BxPC3. Also, we constructed and fitted a dynamical mathematical model to simulate these perturbations and formulate cell line models revealing a striking heterogeneity among them. Predictions of the model respect the effect of GMZ in the accumulation of ceramide in $\mathrm{BxPC3}$ and glucosyl-ceramide in MiaPaca-4 were confirm experimentally. Altogether, these results indicate that fluorescent-SL analogues can be used as sensors of chemotherapy in pancreatic cancer and reveal pathway dynamics between different cell lines, and its potential usage for the development of in vitro chemosensitivy assays able to dissect pathway dynamics to overcome resistance.
\end{abstract}

Keywords-Cancer; GMM; EDO; Sphingolipids.

\section{INTRODUCTION}

Cancer refers to a group of diseases in which cells display uncontrolled growth[1]. Chemotherapy options are usually conditioned by generating resistance which is explained in part by the intrinsic heterogeneity or robustness of the population in the tumor. Biological robustness is recognized as being a conserved organizing principle in biology including adaptation and tolerance, which are enabled by feedback controls, redundancy, modularity and structural stability. Because the use of biosensors for reporting the cellular response to chemotherapy could be achieved at single cell level, we suggest that the dynamic changes on the sphingolipid (SL) signaling pathway could be seen as a biosensor to predict chemotherapy response. This pathway is able to integrate different types of cellular stress signals and report dynamic phenotypes related to induction of cell death pathways [2].
However, the cellular content of different sphingolipids is usually quantified at the whole-cell population level using mass spectrometry techniques. Therefore, we propose to use fluorescently labeled sphingolipid analogues to estimate the SL dynamics at the single cell level. In the present work we sought to investigate the possible application of SM-BOD (fluorescentsphingomyelin analogue) metabolism as a gemcitabine (GMZ) response sensor in pancreatic cancer cells, based on the comparison between GMZ resistant (Panc-1, MiaPaca-2) and sensitive cell lines (BxPC3), which difference was previously reported to be dependent on critical differences in the SLpathway [3]. To evaluate this we loaded cells with SM-BOD and assessed if they display dynamic changes in total cell fluorescence by imaging flow cytometry. In order to analyze the differential SM-BOD cellular metabolism related to resistance or sensitivity, we included known perturbations to the SL pathway in the presence and absence of GMZ. In addition, we extracted multiple image features of fluorescence texture and distribution to create multidimensional data at the single cell level and developed an algorithm based on gaussian-mixture models to study cellular heterogeneity and to generate a perturbation-response fingerprint for each cell line.

In other hand, the SL signaling pathway is in fact complex, with multiple inputs and outputs, and is regulated spatially. Thus a systems level approach is necessary in order to deconvolve this pathway complexity to identify differences related to GMZ resistance. A dynamic mathematical model of SL-pathway was constructed to simulate the total fluorescent level changes upon perturbations. This model was fitted to the experimental, revealing striking differences in the chemotherapy response of the SL-pathway among cell lines.

Altogether, the results indicated that fluorescent SL analogues can report changes related to resistance or sensitivity to chemotherapy in pancreatic cancer and provide information about pathway dynamics at the subpopulation level. This suggests a useful potential of this SL analogues as sensors of chemotherapy response for the development of in vitro chemosensitivy assays able to disentangle cancer heterogeneity. 


\section{MATERIALS AND METHODS}

\section{A. Cell viability and cell death assays}

In order to evaluate the chemosensibility of three pancreatic cancer cell lines to gemcitabine (GMZ), cell viability and cell death assays were stablished using a PrestoBlue assay and propidium iodide (PI) internalization assay, respectively. Different concentrations of GMZ were tested in the cell lines BxPC3 (sensible), MiaPaca-2 and Panc-1 (resistants), which were seeded in 96 well plates for $24 \mathrm{~h}$ and incubated with $72 \mathrm{~h}$ with the chemotherapy.

\section{B. Evaluation of the SM-BOD Metabolism}

A flow cytometry assay was performed for evaluating the cellular metabolism of SM-BOD as a potential sensor for the response to GMZ in the pancreatic cancer lines. Cells were seeded in 24 well plates, loaded with $0.25 \mu \mathrm{M}$ of SM-BOD for 30 min and treated with new medium containing the different inhibitors of the SL-pathway in presence/absent of GMZ (single or double perturbations): an sphingosine kinase inhibitor (SKI, $20 \mu \mathrm{M}$ ), glucosyl-ceramide synthase inhibitor (PDMP, $50 \mu \mathrm{M}$ ), neutral ceramidase inhibitor (NCI, D-erytro-MAPP, $50 \mu \mathrm{M}$ ), acidic ceramidase inhibitor (ACI, C-NMAPPD, $20 \mu \mathrm{M}$ ), ceramide kinase inhibitor (CKI, $50 \mu \mathrm{M})$, a sphingomyelinsynthase inhibitor $(\mathrm{D} 609,100 \mu \mathrm{M})$, an acid sphingomyelinase inhibitor (Desipramine, Des, $25 \mu \mathrm{M}$ ), a ceramide synthase inhibitor (FB1, fumonisin B1, $10 \mu \mathrm{M}$ ), a neutral ceramidase inhibitor (GW, GW4869, $5 \mu \mathrm{M})$ and a serine-palmitoyl CoA reductase (de novo synthesis) inhibitor (Myriocin, Myr, 200 $\mathrm{nM}$ ). After 24h of incubation, acquisition of images was done and they were analyzed using the IDEAS software. Several intensity, texture and morphological features were extracted from the cellular images for further analysis. All the measurements normalized in order to compare the initial values with the remaining fluorescence after $24 \mathrm{~h}$ of exposition.

\section{GMM Algorithm for the analysis of cellular heterogeneity}

From the data obtained with the image analysis of flow cytometry assay, an unsupervised classification was established with Gaussian mixtures algorithm (GMM). This was done in order to create profiles of heterogeneity and identify those conditions that have a similar effect on the distribution of the fluorescence of SM-BOD at cellular level, and thus establish how the GMZ alters or is sensed in the sphingolipids pathway.

\section{Kinetics and predictions of the ODE mathematical model}

In order to infer the transit of the SM-BOD in the sphingolipid pathway, we proceeded with construction of a
A ${ }^{120}$
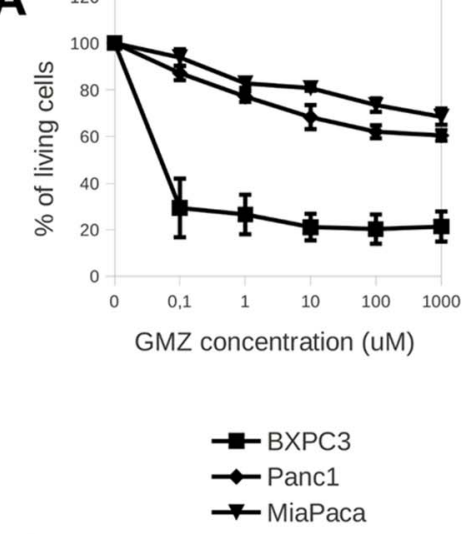

B

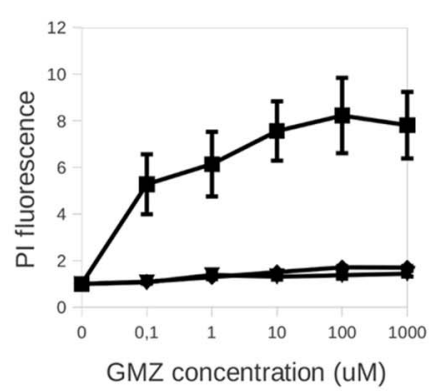

C

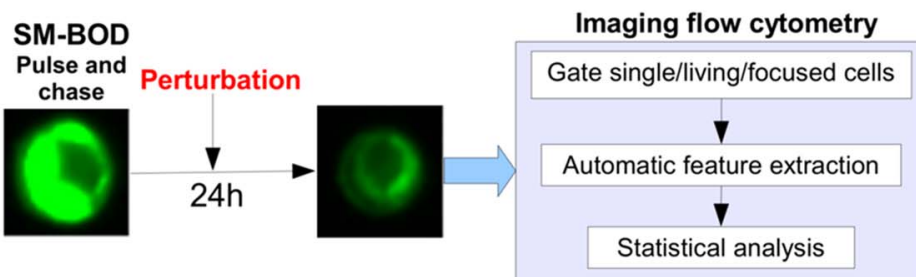

D

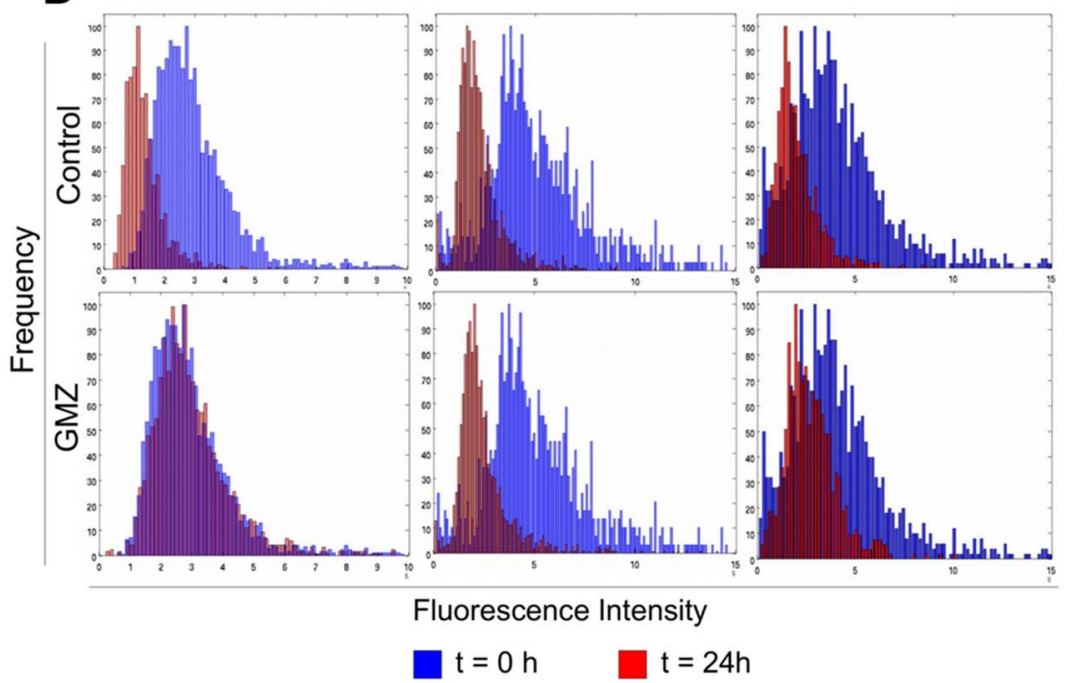

Figure 1. (A) Cell viability and (B) cytotoxicity assays demonstrate that GMZ has a strong cytotoxic activity on the BxPC3 sensible cell line but only a slight antiproliferative activity on the resistant cell lines Panc-1 and MiaPaca-2, being 100 uM the selected concentration that maximizes the difference. (C) An imaging flow cytometry assay was performed for evaluating the cellular metabolism of SM-BOD as a potential sensor for the response to GMZ in the pancreatic cancer lines. All the information of the measurements was extracted for analysis in the models. (D) Intensity distribution of the samples are plotted as histograms, 
mathematical model disturbance-based model, incorporating experimental data and literature information. Subsequently, the reaction kinetics was incorporated using ordinary differential equations (ODE) and based on the law of mass action, Michaelis-Menten kinetics or Hill coefficient. For parameters estimation, data of flow cytometry experiments were incorporated, adjusted separately and independently per cell line and with the Simmulated Anneling algorithm.

With the purpose of determine the predictions of the mathematical model with respect to changes in the composition of sphingolipids by cell line, simulations of presence / absence of GMZ in each model of each line were established, allowing inferring the relative amounts of sphingolipids. For the perturbation with GMZ, it was assumed that chemotherapy had the same effect that the inhibitor where it had been sensed.

\section{E. Validation assay}

The validation of changes in the composition of sphingolipids due to metabolism SM-BOD in the cell lines in presence/absence of GMZ was made using thin layer chromatography (TLC), comparing the BxPC3 cells with both the Panc 1 and MiaPaca-2 cells. Briefly, the lipids were extracted using a mixture of isopropanol:water:ethyl-acetate and then the TLC was run. Pictures were taken under the excitation of a common UV transiluminator. It was possible to obtain a resolution able to separate the fluorescent SL-species of four standards in the order according to their migration: sphingomyelin-BODIPY, glucosyl-ceramide BODIPY, BODIPY-fatty acid and ceramide-BODIPY.

\section{RESULTS}

\section{A. Response to GMZ can be sensed by changes in fluorescent sphingolipid metabolism in pancreatic cancer cell lines}

In order to establish an optimal GMZ concentration for the experimental conditions, we compared the sensitivity of $\mathrm{BxPC} 3$, Panc1 and MiaPaca-2 cells to different concentrations of GMZ. For all cell lines, no significant increase in cell death was observed during the first 48h (not shown). However, the BxPC3 cells were drastically impacted after $72 \mathrm{~h}$. The BxPC3 cells showed a significantly reduced viability to $20-30 \%$ of the control when incubated with GMZ concentrations as low as 0.1 $\mu \mathrm{M}$ (Figure 1A). This decrease in viability was accompanied by a dose-dependent increase in PI labeling, reaching a maximum at a concentration of $100 \mu \mathrm{M}$, indicating that the reduced $\mathrm{BxPC} 3$ cell number is related to cell death (Figure 1B). On the other hand, both Panc1 and MiaPaca-2 cell lines showed only a dosedependent reduction in cell viability to a minimum of $65 \%$ at $100 \mu \mathrm{M}$, without a significant increase in PI staining, suggesting that GMZ induces only an antiproliferative effect on those resistant cell lines. Based on these results, we chose a concentration of $100 \mu \mathrm{M}$ of GMZ (referred as GMZ hereafter) to maximize the effect, if any, of GMZ on both resistant cell lines (Panc1 and MiaPaca-2) compared to the sensitive BxPC3 cell line.
A

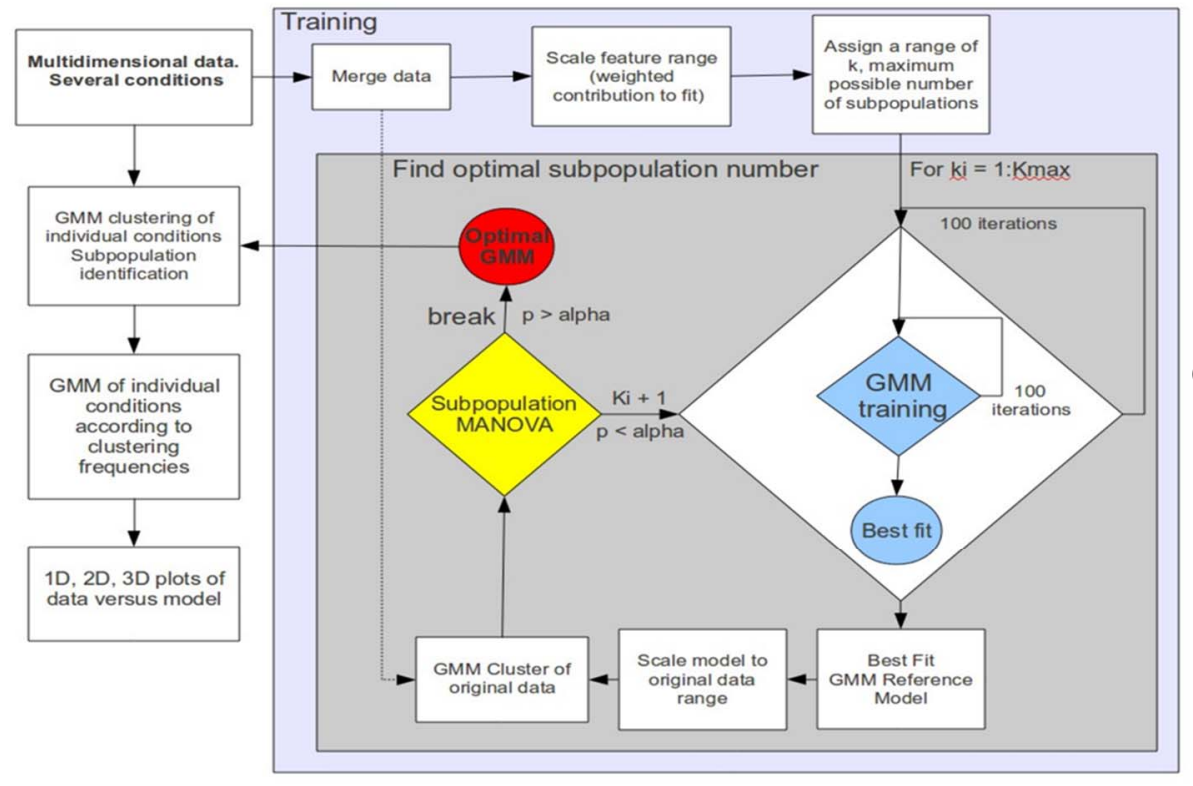

B

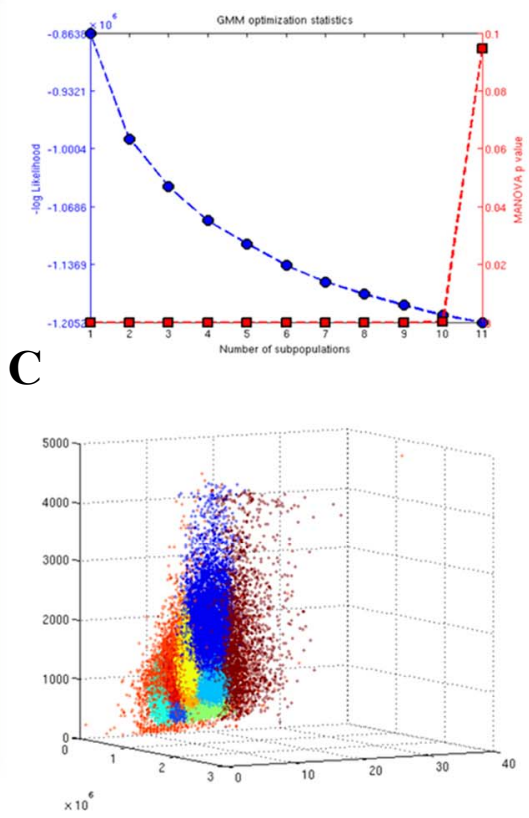

Figure 2. Gaussian Mixure Model. (A) The implementation of a GMM sets a script in MATLAB with three general steps: (i) data from all conditions were combined to form a total population to explore the whole range of possible disturbances, (ii) the number of subpopulations of cells that maintained normal distribution in the entire space of possibilities and n-dimensions (the number of features) were identified, and (iii) each cell was reallocated to its corresponding disturbance assay and a classification was performed with the original cells to divide between subpopulations and then a MANOVA test to determine if that classification identifies subpopulations that differ significantly from each other. The algorithm defines the optimal iteration as that maximum number of Gaussians subpopulations able to identify subpopulations with statistically significant difference (B). Subpopulations in the whole-space of possibilities are shown in (C). 
To evaluate whether the cellular metabolism of SM-BOD has potential as a sensor for the response to chemotherapy (GMZ) in pancreatic cancer, we loaded cells with SM-BOD and assessed if they display changes in the remaining cellular fluorescence after $24 \mathrm{~h}$ compared to control. Cells were seeded and exposed to SM-BOD for $30 \mathrm{~min}$, and after $24 \mathrm{~h}$ incubation, the cells were analyzed by imaging in the flow cytometer. The acquired images were analyzed according to intensity, texture and morphological features (Figure 1C). The distributions of cellular fluorescence intensities (total pixels) showed differences between the sensitive and resistant cell lines (Figure 1D) after 24h.

\section{B. The cellular effects of GMZ can be sensed in the SL- pathway}

A GMM was performed in order to establish a heterogeneity profile per each perturbation as a fingerprint (algorithm details in Figure 2-A). For this propose, the data of cell features in each perturbation assay were joined for identifying the maximum number of subpopulations with normal distribution and evaluated with a MANOVA test. Iteratively, it was concluded that the maximum number of subpopulations was 10, as it is shown in Figure 2-B. The whole space of possibilities is presented in Figure 2-C where is possible to identify the 10 gaussian subpopulations. In the algorithm, the fingerprint in Figure 3 (left) was obtained from reclassify all the cells into their original assay and then determinate the frequency of each subpopulation in the perturbation. Finally, the clustering analysis to evaluate the similarities of the heterogeneity profile was made with a statistic significant of 0.05 and Euler distance, where is possible to identify that D609 and GMZ have similar profile, suggesting that modification of the sphingolipids pathway of both remains close and concluding that GMZ can be sensed in the step where D609 acts, the Sphingomyelin-Synthase (Figure 3 , right).

\section{Predictions of the ODE model about the composition of SL in BxPC3 and MiaPaca-2 with GMZ treatment can be verified experimentally}

The topological model of the pathway of sphingolipids based on available data and literature is presented in Figure 4A. The topology was made with iterative changes based on minimize the adjustment function. The model starts with an input of SM-BOD (sphingomyelin-BODIPY) assumed 100\% at time 0 , and which is metabolized by different enzymes and transformed into various species that may or may not preserve the fluorescent species.. The parameter estimation was performed with each cell line and data of the perturbations were incorporated into each model in both presence/ absence of GMZ. Figure 4-B presents the comparison of total fluorescence of experimental and simulated data for cell lines in the absence of chemotherapy, which shows that the model does fit the experimental data (with an acceptable error less than one standard deviation of the experiments). Furthermore, it can be seen that the total fluorescence (and therefore the composition sphingolipid) in each cell line is different. To infer the

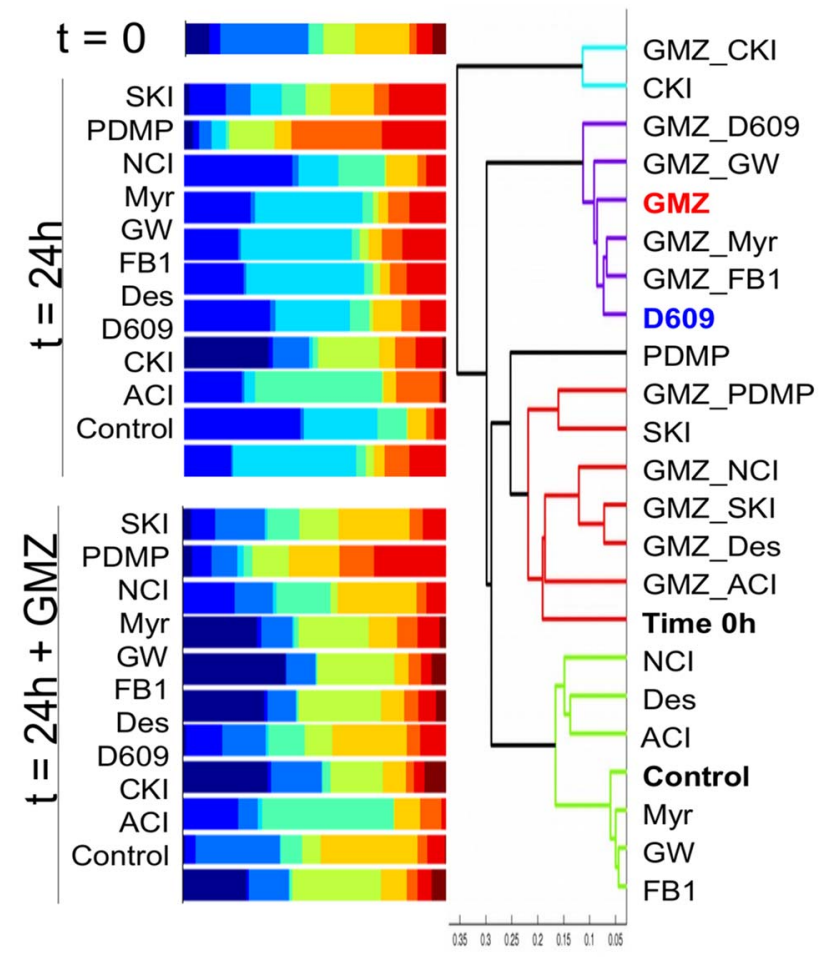

Figure 3. Clustering analysis. Using the GMM, data of each disturbance (inhibitor with/without GMZ) and the frequency of each subpopulation define a pattern of heterogeneity (fingerprint) related to each disturbance. With the fingerprint information, a clustering analysis to associate the various disturbances each other was made. Clustering shows that GMZ can be sensed as the D609 inhibitor.

composition of sphingolipids in each case, simulations in the presence / absence of GMZ, were conducted as it is shown in Figure 4-C, revealing a significant increase of ceramide in BxPC3 and glucosyl-ceramide in MiaPaca-2 cell lines under exposition to GMZ, which are set as hypothesis of the mathematical model.

In order to validate the changes obtained from the mathematical model of different fluorescence intensities between sensitive and resistant cell lines in SL-sensor metabolism upon GMZ, we studied the fluorescent-SL composition of cell pellets using thin layer chromatography (TLC) comparing the BxPC3 cells with both the Panc1 and MiaPaca- 2 cells. The BxPC3 cells showed a striking increase of ceramide-BODIPY and a slight increase in BODIPY-labeled fatty acid upon GMZ treatment compared to control. On the other hand, Panc-1 cells showed no significant differences in lipid composition upon treatment, whereas MiaPaca-2 cells showed a shifted band closed to the glucosyl-ceramideBODIPY. This indicates that GMZ alters the SL-metabolism deviating it towards the production of mainly ceramide in the GMZ sensitive BxPC3 cells. In contrast, GMZ has no effect on the SL metabolism in Panc-1 cells. 

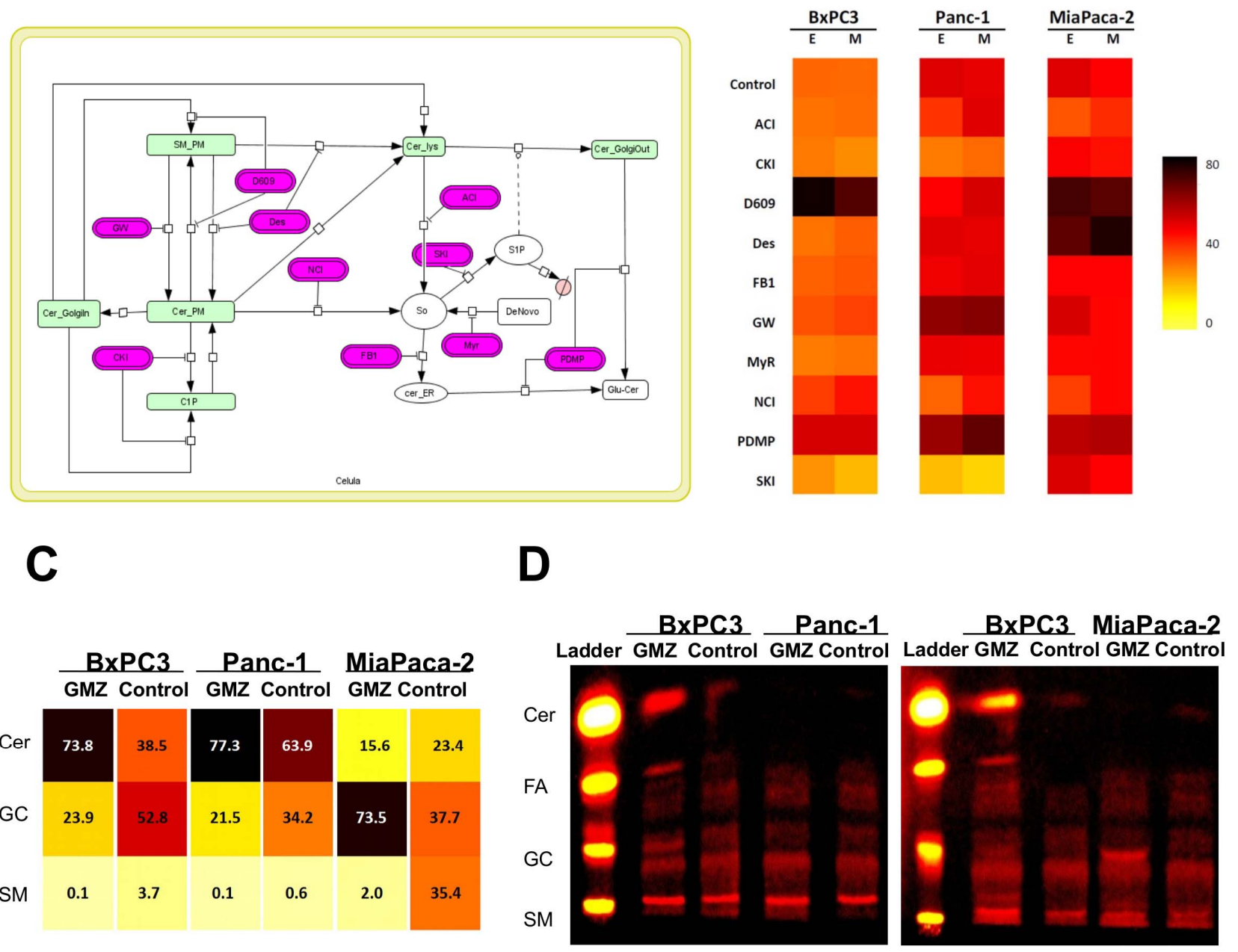

Figure 4. ODE mathematical model of SL. (A) Topology of the model shows the transit of the SM-BOD in different compartments. Some reactions can ends in a loss of the fluorescence specie of the SL-analogue and consequently a reduction of the total fluorescence. The parameters estimation was done with the single or double perturbation data, where it is possible to appreciate the fitted model and a cell line specificity of the total fluorescence in each condition (B, only for single perturbation). Predictions of the model were run for both control and GMZ conditions, showing a different distribution of SL in all the cell lines (C). Validation of those changes was verified experimentally with a TLC assay, showing accumulation of ceramide(Cer) in the BxPC3 and glucosyl-ceramide (GC) in MiaPaca-2 in presence of GMZ.

Interestingly, GMZ produces changes in the SL metabolism in MiaPaca-2 cells which are reflected only as a slight shift in fluorescence intensity compared to control (Figure 1D), although they are not related to cell death.

\section{DISCUSSION}

Because the role of sphingolipids in cell signaling and the mechanisms of death/ survival, cancer approach with the use of sphingolipids pathway provides the ability to seek new alternatives for customization of therapy. In our study, the characterization of the cell lines according to their sensitivity to GMZ was described with viability and cytotoxicity assays, confirming the sensitivity of BxPC3 and resistance of Panc-1 and MiaPaca-2. In addition, changes in the composition of sphingolipids, in the presence and absence both of GMZ, was showed with the SM-BOD and total fluorescence values.

In order to gain insight into the effect of GMZ on the SL metabolism of each cell line, we designed a strategy of single and double perturbations to identify interactions between GMZ and known perturbations to the SL pathway. The interactions of GMZ with the SL-pathway perturbations also differ between the cell lines which suggest the presence of underlying cell linespecific differences in the SL-metabolism. Furthermore, this response profiles did not seem homogeneous even within the cells of the same cell line. This feature was considered in a study conducted in 2008, which it showed the heterogeneity role with data of biosensors in a GMM model. In this case, analysis of GMM was used to characterize cellular phenotypes in drug response, allowing to distinguish different classes of drugs based 
on its mechanism of action and revealing biological differences in populations selected by the GMM [4].

In our case, a model of GMM was performed with several morphological, intensity and texture features which were extracted from the cellular images of the flow cytometry assay, showing the differences in the subpopulation composition as a fingerprint. According to the clustering analysis of the profiles in each condition of our study, the GMM shows that D609 and GMZ have similar profile, suggesting that GMZ can be sensed in the step where D609 acts, the Sphingomyelin-Synthase, which has not been previously reported. In fact, there are few reports in the literature about how chemotherapies can be sensed in the sphingolipid pathway. Doxorubicin and etoposide for example affect the function of sphingosine kinase [5].

In other hand and using a system biology approach, an ODE model was fitted with the fluorescence data of the perturbations, including the effect of the chemotherapeutic drug GMZ in the Sphingomyelin-Synthase. The proposed model of sphingolipids simulated the transit of the SM-BOD fluorescent species for the cell. Since the proposed model was based on disturbances and ordinary differential equations, it was possible to describe the effect or impact of the GMZ in specific SL (by inference) after it has reached a state of equilibrium, which served to generate hypothesis. Predictions of the mathematical model reveal an accumulation of ceramide in sensible cell line BxPC3 in presence of GMZ, which were shown with the experiment result with the TLC assay.

In this regard, sphingolipids play a key role in activation of death mechanisms, being ceramide one of the most characterized species, which is involved in apoptotic, necroptosis and autophagy death mechanisms and occurs in various cellular compartments[6]. For the simulation of Panc-1 cell line, the simulations show some discrete changes in ceramide and glucosyl-ceramide, which could not being verified with the TLC assay, however, in the case of MiaPaca-2, the accumulation of glucosyl-ceramide was predicted by the mathematical model and clearly shown in the validation assay.

Several reports have shown a relationship between the activity of the glucosyl ceramide synthase (GCS) and chemoresistance, in which multiple mechanisms may be involved, including decreased the concentration of biologically active ceramide [6]. Giussani and coworkers showed that glucosylceramide protects glioblastoma cells against autophagic and apoptotic by Temozolomide and Paclitaxel induced death, and the concomitant use of inhibitors of glucosylceramide synthase revert that resistance to enhance the cytotoxic effects of these drugs [7]. These two reports are consistent with experimental data MiaPaca-2 cell line.

However, a 2003 study in which evaluated Doxorubicin, Paclitaxel, Cytosine arabinoside, and analogs of ceramide of short chain did not generate difference in sensitivity of a line of murine melanoma by inhibiting GCS or increase their activity [8]. These findings suggest that the regulation of GCS in chemoresistance depends on the type of cell that can act through different mechanisms, which could explain the few changes seen for the Panc-1 cell line.
In general, the usage of double perturbation studies lets to understand the interactions of single components and systems level properties, which is wealthy for understanding the biology of SL in the cell cancer in this case. Taken together, these results demonstrate the potential of the SL-sensor to report changes upon perturbations in SL-metabolism at the population level induced by the chemotherapeutic drug GMZ, which affect the Sphingomyelin-Synthase activity. They also indicate that although GMZ does not induce cell death in resistant cell lines, they in fact respond to GMZ by changes in their SL-metabolism which are more evident in the presence of specific perturbations. Cell specific responses could be related with resistance and therefore the understanding of these responses has a potential for the design of strategies to revert drug resistance.

\section{ACKNOWLEDGMENT}

We thank the valuable contributions of Dr. Stefan Legewie and Professor Roland Eils.

\section{REFERENCES}

[6] L. Chai, R. P. McLaren, A. Byrne, W.-L. Chuang, Y. Huang, M. R. Dufault, J. Pacheco, S. Madhiwalla, X. Zhang, M. Zhang, B. a Teicher, K. Carter, S. H. Cheng, J. P. Leonard, Y. Xiang, M. Vasconcelles, M. a Goldberg, D. P. Copeland, K. W. Klinger, J. Lillie, S. L. Madden, y Y. a Jiang, «The chemosensitizing activity of inhibitors of glucosylceramide synthase is mediated primarily through modulation of P-gp function.», Int. J. Oncol., vol. 38, n. ${ }^{\circ}$ 3, pp. 701-11, mar. 2011.

[7] P. Giussani, R. Bassi, V. Anelli, L. Brioschi, F. De Zen, E. Riccitelli, M. Caroli, R. Campanella, S. M. Gaini, P. Viani, y L. Riboni, «Glucosylceramide Synthase Protects Glioblastoma Cells Against Autophagic and Apoptotic Death Induced by Temozolomide and Paclitaxel», Cancer Invest., vol. 30, n. ${ }^{\circ}$ 1, pp. 27-37, ene. 2012.

[8] R. J. Veldman, A. Mita, O. Cuvillier, V. Garcia, K. Klappe, J. A. Medin, J. D. Campbell, S. Carpentier, J. W. Kok, y T. Levade, «The absence of functional glucosylceramide synthase does not sensitize melanoma cells for anticancer drugs.», FASEB J., vol. 17, n. ${ }^{\circ}$ 9, pp. 1144-6, jun. 2003. 